\title{
Woven thermoplastic composite forming simulation with solid-shell element method
}

\author{
Q.Q. Chen ${ }^{1}$, A. Saouab ${ }^{1, a}$, P. Boisse ${ }^{2}$, C.H. Park ${ }^{1}$, J. Bréard ${ }^{1}$ \\ ${ }^{1}$ Laboratoire Ondes et Milieux Complexes, FRE 3102, CNRS, Université du Havre, France \\ ${ }^{2}$ Laboratoire de Mécanique des Contacts et des Structures, UMR 5259, CNRS, INSA Lyon, France
}

Received 17 October 2008, Accepted 04 April 2009

\begin{abstract}
Textile composites become more and more popular in aeronautic industries, due to their high performances. In this work, based on the model shown in the paper [9], a solid-shell element is used to simulate the woven composite forming. Comparing with other shell elements, a distinctive advantage of solid-shell elements is that the complication on handing finite rotations does not exist. Accounting the specific mechanical behaviors of woven composite material, the tensile, in-plane shear, bending and compressive energies are taken into account depending on the fiber direction, and then the total strain energy is computed to be the sum of those energies. The necessary material data for the simulation come from standard tensile, compressive and bias test experiments. Some forming simulations are performed with this method, and we can observe the wrinkles that exhibit the influence of bending stiffness. The results show the efficiency of the approach.
\end{abstract}

Key words: Solid-shell element, Woven thermoplastic composite, forming simulation

\section{Introduction}

Woven composites are now gaining the popularity in manufacturing automotive and aerospace parts due to their high mechanical performances. This calls for a development of adequate design and analysis methods and reliable simulation tools considering the large deformations of textile reinforcements in composite forming process. Indeed, during the forming stage, the possible deformation modes of the composite are those of the reinforcement. The woven reinforcement behaviors are very special. The tensile stiffness plays a major role in the fiber direction, and the others stiffnesses, such as the in-plane shear, bending and compressive stiffnesses are very small compared to it. Even so, they are important in some simulation. For example, the in-plane shear stiffness causes the wrinkles and the bending stiffness can change the form of wrinkle. The compressive stiffness can't be ignored in the consolidation and injection case.

For modeling the performing of woven reinforcements, two different approaches exist: the geometrical approach and the finite element approach. The models based on the geometrical approach are called fishnet algorithms [1-2]. An alternative to these geometrical methods is the use of finite element approaches. Some of them are obtained by homogenizing the mechanical behavior of the underlying meso-structure and considering the fabric as an anisotropic continuum [3-4]. Conversely, some discrete models are also developed for fabric in which each yarn is modeled and is assumed to be a straight or a curved beam or truss [5]. And other authors have proposed the semi-discrete element methods [6-8]. All those models have been proved to be efficient and fast in the suitable cases. However, we note that the bending and compressive stiffness are rarely considered in those models.

In the present work, solid-shell element model has been proposed for the simulation of fabric shaping process, which is based on a meso-macro approach. This type of element has only the translational but no rotational nodal degree of freedoms. Fabric forming is a typical geometric nonlinear problem, in which the strains in the yarn directions are small (less than $2 \%$ for the glass fabric), while the displacement and the rotation are large. Thus, the strain energy is considered in the field of large strain kinematics. The tensile, bending and shear energies can be expressed as simple functions of the nodal displacements, with the explicit method. Subsequently, we can obtain the internal force in function of the nodal displacements.

\section{Solid-shell element}

In this section, the formulation of the solid-shell element is discussed.

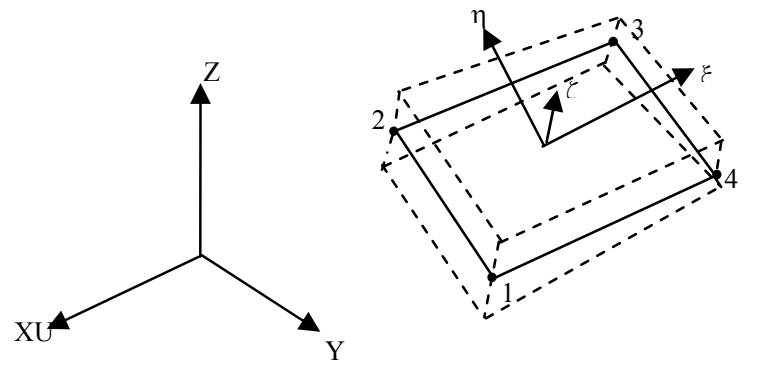

Fig 1 Solid-shell element

With reference to the solid-element in figure 1, the undeformed position vector $\mathrm{X}$ and the displacement vector $\mathrm{U}$ can be written as:

\footnotetext{
${ }^{a}$ Corresponding author: abdelghani.saouab@univ-lehavre.fr
} 
$X(\varepsilon, \eta, \zeta)=X_{o}(\varepsilon, \eta)+\zeta X_{n}(\varepsilon, \eta)=\sum_{i=1}^{4} N_{i}(\varepsilon, \eta) X_{o i}+\zeta \sum_{i=1}^{4} N_{i}(\varepsilon, \eta) X_{n i}$

$U(\varepsilon, \eta, \zeta)=U_{o}(\varepsilon, \eta)+\zeta U_{n}(\varepsilon, \eta)=\sum_{i=1}^{4} N_{i}(\varepsilon, \eta)\left(U_{o i}+\zeta U_{n i}\right)=\left[\begin{array}{ll}N & \zeta N\end{array}\right] u$

where $X_{o}=\left.X\right|_{\zeta=0,} U_{o}=\left.U\right|_{\zeta=0}, X_{n}=\left.X\right|_{\zeta=+h / 2}-\left.X\right|_{\zeta=-h / 2}$, $U_{n}=\left.U\right|_{\zeta=+h / 2}-\left.U\right|_{\zeta=-h / 2}$. The interpolation function is:

$$
\begin{aligned}
\{N\}=\left\{\begin{array}{l}
N_{1}(\varepsilon, \eta) \\
N_{2}(\varepsilon, \eta) \\
N_{3}(\varepsilon, \eta) \\
N_{4}(\varepsilon, \eta)
\end{array}\right\}=\frac{1}{4}\left\{\left(\left\{\begin{array}{l}
1 \\
1 \\
1 \\
1
\end{array}\right\}+\varepsilon\left\{\begin{array}{c}
-1 \\
1 \\
1 \\
-1
\end{array}\right\}+\eta\left\{\begin{array}{c}
-1 \\
-1 \\
1 \\
1
\end{array}\right\}+\varepsilon \eta\left\{\begin{array}{c}
1 \\
-1 \\
1 \\
-1
\end{array}\right\}\right)\right. \\
\mathrm{N}=\left[\begin{array}{llll}
I N_{1} & I N_{2} & I N_{3} & I N_{4}
\end{array}\right]
\end{aligned}
$$

It can be seen that the undeformed and deformed nodal directors are $X_{n i}$ and $X_{n i}+U_{n i}$ respectively. $\zeta=0$ represents the mid-surface and $X_{n i}$ is of unit length. The natural Green strain is:

$$
\varepsilon_{i j}=\varepsilon_{i j}(U)=\left(\bar{X}_{, i}^{T} \cdot \bar{U}_{, j}+\bar{X}_{, j}^{T} \cdot \bar{U}_{, i}\right) / 2+\left(\bar{U}_{, i}^{T} \cdot \bar{U}_{, j}+\bar{U}_{, j}^{T} \cdot \bar{U}_{, i}\right) / 4
$$

Then the in-plane strain can be written as:

$$
\varepsilon_{i j}=\varepsilon_{i j}^{(0)}+\xi \varepsilon_{i j}^{(1)}+\xi^{2} \varepsilon_{i j}^{(2)}
$$

where $i, j=\zeta, \eta$. In the in-plane natural strains, the zeroth and first-order $\zeta$-terms are commonly known as the membrane and bending strains, respectively. The second-order $z$-terms are dropped. Thus, equation (5) can be simplified as:

$$
\varepsilon_{i j}=\varepsilon_{i j}^{m}+\varepsilon_{i j}^{b}
$$

With equation (3), we can express the bending strains and the membrane strains respectively:

$$
\begin{gathered}
\varepsilon_{i j}^{b}=\varsigma\left(\bar{X}_{n, i}^{T} \cdot \bar{U}_{o, j}+\bar{X}_{o, i}^{T} \cdot \bar{U}_{n, j}+\bar{X}_{n, j}^{T} \cdot \bar{U}_{o, i}+\bar{X}_{o, j}^{T} \cdot \bar{U}_{n, i}\right) / 2 \\
+\varsigma\left(\bar{U}_{o, i}^{T} \cdot \bar{U}_{n, j}+\bar{U}_{o, j}^{T} \cdot \bar{U}_{o, i}+\bar{U}_{n, i}^{T} \cdot \bar{U}_{o, j}+\bar{U}_{n, j}^{T} \cdot \bar{U}_{o, i}\right) / 4 \\
\varepsilon^{m}{ }_{i j}=\left(\bar{X}_{o, i}^{T} \cdot \bar{U}_{o, j}+\bar{X}_{o, j}^{T} \cdot \bar{U}_{o, i}\right) / 2 \\
+\left(\bar{U}_{o, i}^{T} \cdot \bar{U}_{o, j}+\bar{U}_{o, j}^{T} \cdot \bar{U}_{o, i}\right) / 4
\end{gathered}
$$

We can also define the compressive normal strain as:

$\varepsilon_{\zeta \varsigma}=\left.N_{1} \varepsilon_{\zeta \varsigma}\right|_{\varepsilon=-1, \eta=-1}+\left.N_{2} \varepsilon_{\zeta \zeta}\right|_{\varepsilon=1, \eta=-1}+\left.N_{3} \varepsilon_{\zeta \varsigma}\right|_{\varepsilon=1, \eta=1}+\left.N_{4} \varepsilon_{\zeta \varsigma}\right|_{\varepsilon=-1, \eta=1}$

\section{Dynamic equation}

Usually, the forming process can be considered as the quasi-static case, and the majority of codes for the material forming simulations are based on the explicit dynamic approach:

$$
[M]\{\ddot{U}\}+[C]\{\dot{U}\}+\left\{F_{\text {int }}\right\}=\left\{F_{\text {ext }}\right\}
$$

The textile geometry is particular. It consists of the yarns that intertwined with each other, and the yarns are made of the fiber filaments with very small diameter ( 5 to $7 \mu \mathrm{m}$ for carbon, $10-20 \mu \mathrm{m}$ for aramid, 5-25 $\mu \mathrm{m}$ for glass). The particular geometry results in the particular mechanical behaviors. During the forming process, we can consider that the yarn has the tensile stiffness only in the fiber direction. In some types of analysis, however, it is also noted that it is necessary to take into account the other stiffnesses, because they play a significant role (for example, it is shown that the main stiffening effect due to shear makes the wrinkles when the shear limit angle is surpassed, and the bending stiffness can change the wrinkle form). Hence, in order to obtain the total internal virtual work, we consider the internal virtual works of the warp and the weft caused by tensile loads, bending moment, compressive force as well as by shear.

$$
\begin{aligned}
& -W_{\text {int }}=\sum\left(\varepsilon^{m}{ }_{\text {warp }}(\bar{u}) \sigma^{m}{ }_{\text {warp }}+\varepsilon^{m}{ }_{\text {weft }}(\bar{u}) \sigma^{m}{ }_{\text {weft }}+\gamma(\bar{u}) C_{y}\right. \\
& \left.+\varepsilon^{m}{ }_{\text {warp }}(\bar{u}) \sigma^{m}{ }_{\text {warp }}+\varepsilon^{b}{ }_{\text {weft }}(\bar{u}) \sigma^{b}{ }_{\text {weft }}+\varepsilon_{\zeta \zeta}(\bar{u}) \sigma_{\zeta \zeta}\right)
\end{aligned}
$$

\subsection{Internal work of tension}

To calculate the internal work caused by tension, we should calculate the strain of yarn. We take a unit yarn to analyze, the strain of this unit yarn can be described as:

$$
\varepsilon=\frac{(d s-d S)}{d S}=\frac{d X^{T} E d X}{2 d S^{2}}
$$

Here $\varepsilon$ is the Green strain tensor, $d X$ is the initial direction of yarn, and $d S$ is the initial length of unit yarn, and $d s$ is the length of unit yarn after the deformation.

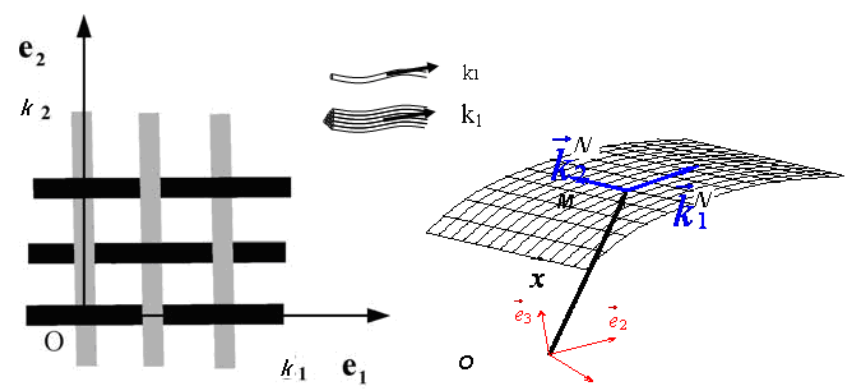

Fig 2: Schematic of the coordinate system

We define a global coordinate frame $\left\{e_{1}, e_{2}, e_{3}\right\}$ (see figure 2 ), in which the initial yarn directions can be represented as:

$$
\bar{k}_{i}=r_{i 1} \bar{e}_{1}+r_{i 2} \bar{e}_{2}
$$

Then, the strain of yarn can be obtained by a following relation.

$$
\varepsilon^{T}{ }_{i i}=\varepsilon^{m}{ }_{11} r_{i 1}^{2}+\varepsilon^{m}{ }_{22} r_{i 2}^{2}+2 \varepsilon^{m}{ }_{12} r_{i 1} r_{i 2}
$$

A number of experiments have proved that the yarns can be assumed to be elastic. The stress caused by tensile loads is expressed as: 


$$
\sigma_{i i}=E \varepsilon_{i i}
$$

where $E$ is the Young's modulus of yarn. Hence, the internal work caused by tensile load can be obtained as:

$$
\begin{aligned}
& -W^{m}{ }_{\text {int }}=\sum\left(\varepsilon^{m}{ }_{\text {warp }}(\bar{u}) \sigma^{m}{ }_{\text {warp }}+\varepsilon^{m}{ }_{\text {weft }}(\bar{u}) \sigma^{m}{ }_{\text {weft }}\right) \\
& =\sum\left(\varepsilon^{m}{ }_{\text {warp }} E_{\text {warp }} \varepsilon^{m}{ }_{\text {warp }}+\varepsilon^{m}{ }_{\text {weft }} E_{\text {weft }} \varepsilon^{m}{ }_{\text {weft }}\right)
\end{aligned}
$$

\subsection{Internal work of shear}

The experimental observations of the shear tests of the woven reinforcements have shown that the fabric deformation is the result of a relative rotation between the warp and the weft yarns but not of a fiber shearing. The relative rotations of the warp and the weft yarns lead to a torque applied at the yarn intersections. The value of this shear torque for a shear angle is experimentally obtained by the bias-test.

The increment of shear strain $\Delta \gamma_{i}$ is assumed to be the sum of the increment of weft and warp rotations.

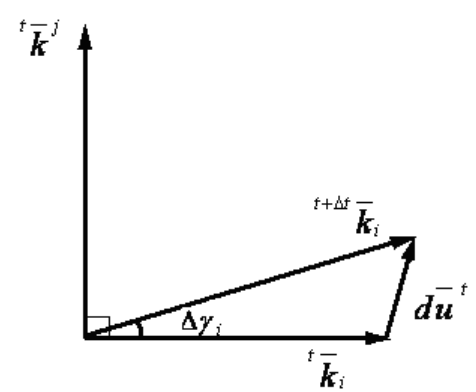

Fig 3: Yarn's shear strain increment

To calculate the yarn shear strain increment, we suppose that $\Delta \gamma_{i}$ is small (see figure 3). Then we can consider:

$$
\Delta \gamma=\sin (\Delta \gamma)
$$

With the geometry shown in figure 3 , we can derive:

$\sin (\Delta \gamma)=\frac{{ }^{t+\Delta t} \bar{k}_{i} \cdot \bar{k}^{j}}{\left\|{ }^{t+\Delta t} \bar{k}_{i}\right\|{ }^{t} \bar{k}^{j} \|}=\frac{d \bar{u} \cdot \bar{k}^{j}}{\left\|{ }^{t+\Delta t} \bar{k}_{i}\right\|\left\|{ }^{t} \bar{k}^{j}\right\|} \cong \frac{d \bar{u} \cdot \bar{k}^{j}}{\left\|\Delta t \bar{k}_{i}\right\|\left\|^{t} \bar{k}^{j}\right\|}$

where $i \neq j$. Then, we obtain:

$$
\Delta \gamma_{i}=\sin \left(\Delta \gamma_{i}\right)=\left(\frac{\partial \Delta \bar{u}}{\partial X_{1}} \cdot \bar{k}^{j}\right) r_{i 1}+\left(\frac{\partial \Delta \bar{u}}{\partial X_{2}} \cdot \bar{k}^{j}\right) r_{i 2}
$$

Since $\Delta \gamma_{i}$ is the shear strain increment of one yarn for the fabric, the shear strain is :

$$
\Delta \gamma=\Delta \gamma_{1}+\Delta \gamma_{2}
$$

The internal work caused by shear can be defined as:

$$
-W_{\text {int }}=\sum\left(\gamma(\bar{u}) C_{y}\right)
$$

where $C_{y}$ is the shear torque which is the function of $\gamma_{i}$ and is obtained by a bias-test.

\subsection{Internal work of bending}

To obtain the internal work of bending, we should find out the bending strains of fabric. In equation (8), we can obtain the bending strains. With the similar relation as equation (14), we can obtain:

$$
\varepsilon^{b}{ }_{i i}=\varepsilon^{b}{ }_{11} r_{i 1}^{2}+\varepsilon^{b}{ }_{22} r_{i 2}^{2}+2 \varepsilon_{12}^{b} r_{i 1} r_{i 2}
$$

Then, with the bending stiffness, we can easily obtain the internal work of bending

$$
-W^{b}{ }_{\text {int }}=\sum\left(\varepsilon^{b}{ }_{\text {warp }} C^{b}{ }_{\text {warp }} \varepsilon^{b}{ }_{\text {warp }}+\varepsilon^{m}{ }_{\text {weft }} C^{b}{ }_{\text {weft }} \varepsilon^{b}{ }_{\text {weft }}\right)
$$

\subsection{Internal work of compression}

We have already presented the compressive normal strain in equation (9). If we obtain the compressive stress, we obtain the internal work of compression. The experiment shows that the relation between compressive strain and compressive stress is nonlinear. Thus, in this paper, the compressive stress is express as a function of the compressive strain, in form of polynomial as:

$$
\sigma^{c}=111.17 \varepsilon_{c}^{3}-26.862 \varepsilon_{c}^{2}+2.683 \varepsilon_{c}+0.0078
$$

Then we obtain the internal work of compression:

$$
-W^{c}{ }_{\mathrm{int}}=\sum\left(\varepsilon^{c} \sigma^{c}\right)
$$

\section{Model validations}

Some validations are presented in this section. Those tests come from the special behaviors of fabric, and usually are used to verify the validity of the model for fabric simulation.

\subsection{Picture frame test}

The picture frame test is conducted to consider a pure shear case. It offers a direct method to measure the response of woven yarns in a deformation mode. In this case, a hinged frame with four equal bars is assembled in a tensile testing machine. A tensile force is applied across diagonally opposing corners of the picture frame rig causing the picture frame to move from an initially square configuration into a rhomboid, like figure 4 . As in-plane shear causes the shear stress only, the stresses in the yarn directions must be zero in the case of simple shear of woven fabric. The result of picture frame test is shown in figure 5 .
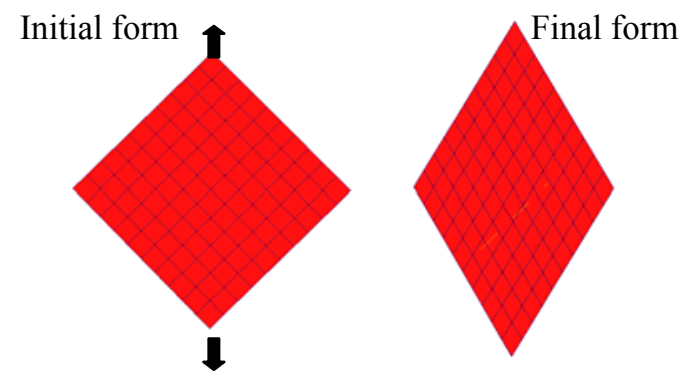

Fig 4: Simulation of picture frame 


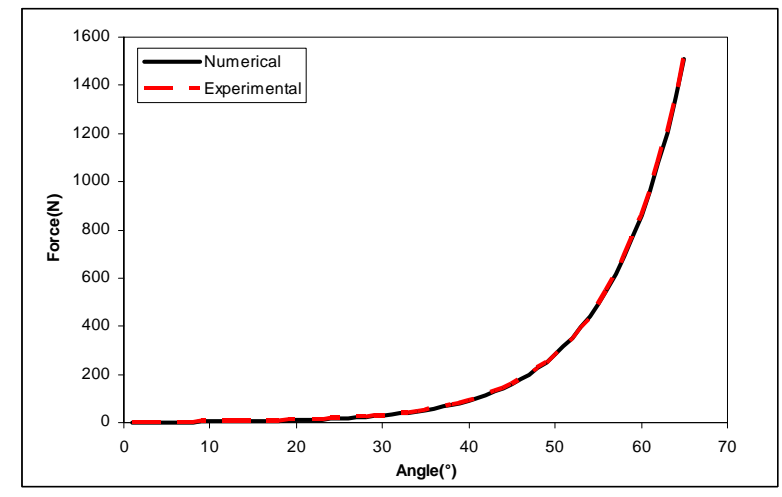

Fig 5: Diagonal force vs shear angle

\subsection{Bias test}

Bias extension test is performed with a fabric sample of a length normally twice greater than the size of width where the fibres are oriented at $45^{\circ}$ to the loading direction (see figure 6). During this test, the central area is sheared whereas four others areas are half-sheared and the clamping areas are not sheared. This test allows to consider the second order stiffness contributions and to determine the continuous material parameters. We can see the result of bias test in figure 7.

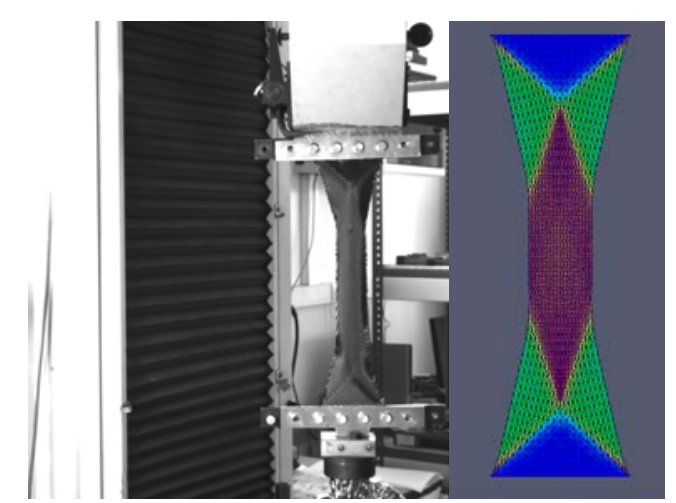

Fig6: Experimental and simulation results of bias test

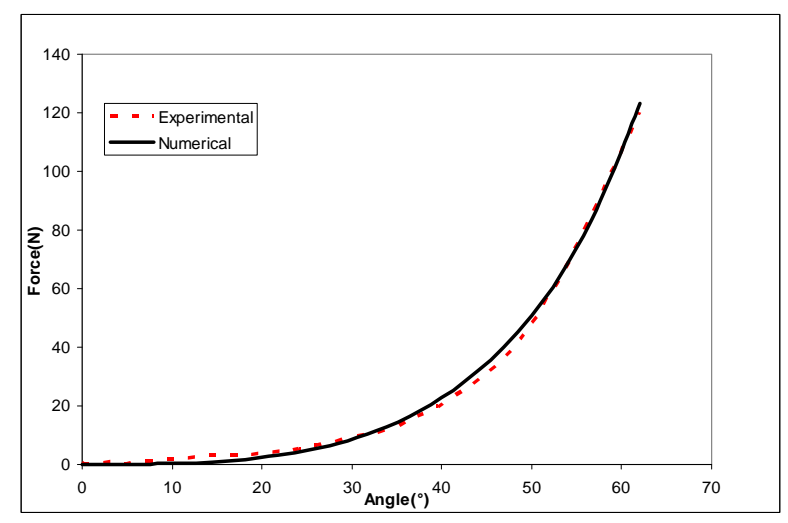

Fig 7: Tensile force vs shear angle

\subsection{Compression test}

An experimental device developed at the University of Le Havre (figure 8) is used for the compression test. Twenty layers woven fabrics which have the same fiber direction are tested by this machine. We use the displacement control and outputs are the compressive normal strain and the normal compressive stress (figure 9).

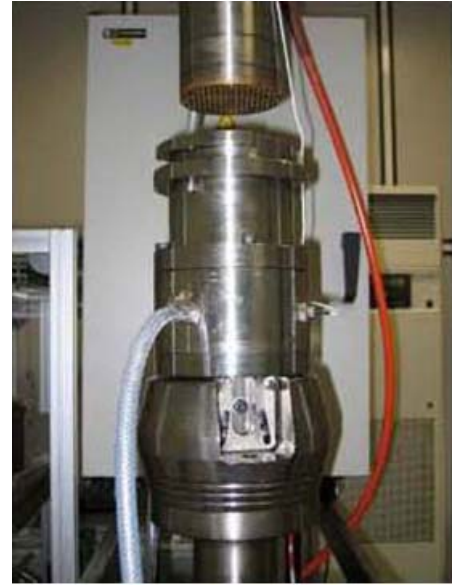

Fig 8: Experimental setup for compression test

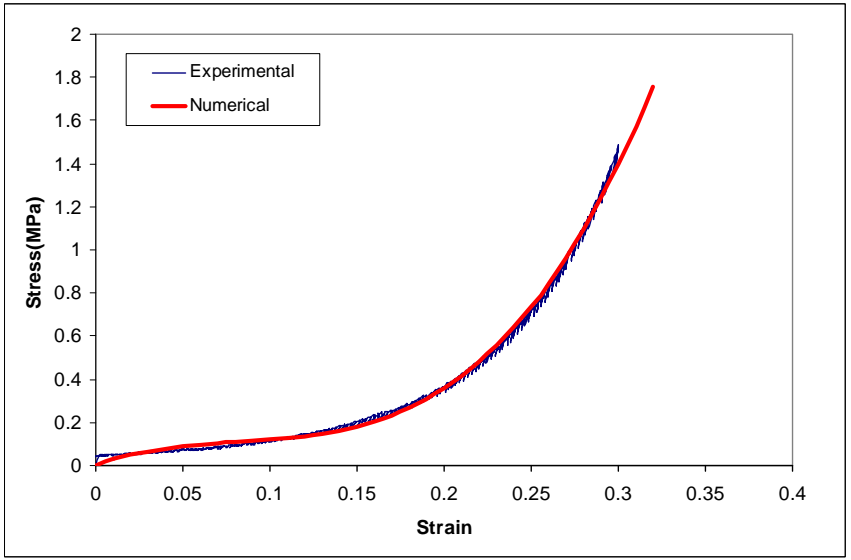

Fig 9: Normal stress vs normal strain

\section{Simulation results}

\subsection{Hemisphere forming simulation}

Hemisphere forming is a classical forming simulation test. From this test, we can observe that the final form is different if the fibre direction is different. Figure 10 and figure 11 show the simulation results of this test, in which the fibre direction is $-45 / 45$. The result show us the change of angle between weft and wrap (figure 10), and the normal strain (figure 11).

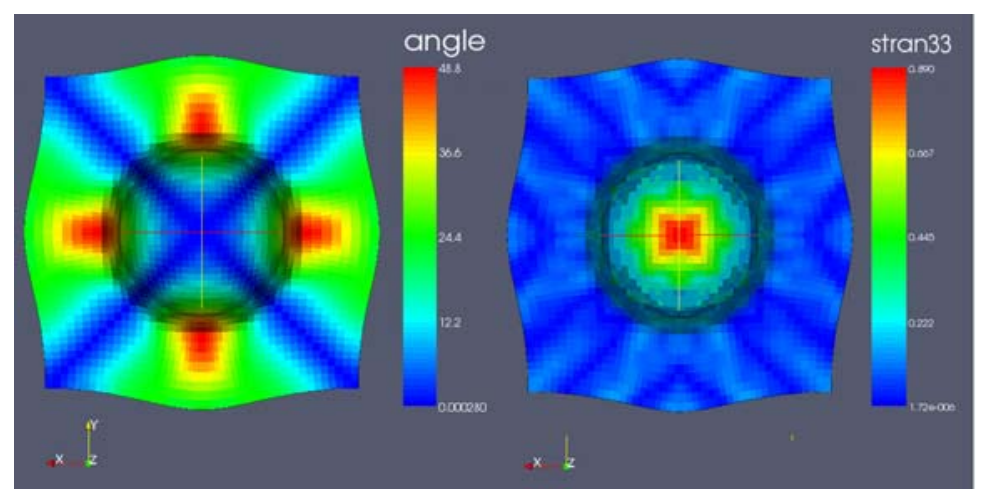

Fig 10: Change of angle

Fig 11: Normal strain

\subsection{Square box deep drawing simulation}

Square box deep drawing simulations have been conducted real woven sheet forming of this geometry is difficult, because it leads to large variations of angle between warp and 
weft in the radius. Three types of simulation have been done with the consideration of the tension only and of both the tension and the shear, finally of the bending as well. Figure 12 presents three deformed shapes of the woven fabric at the end of the forming. There is no wrinkling in the tensile only solution because there is no source of instability in this case. On the other hand, some wrinkles are developed in the consideration of both the tension and the shear. The wrinkles are caused by the fabric shear locking that leads to out-of-plane deformation. We can observe that the form of wrinkle changes if the bending stiffness is also considered.

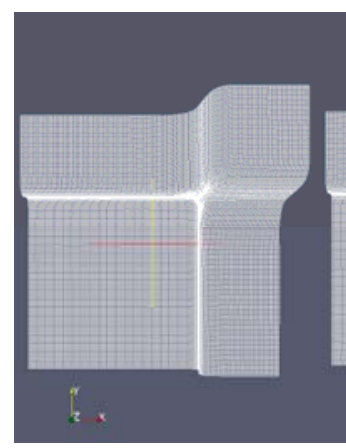

Without shear without bending

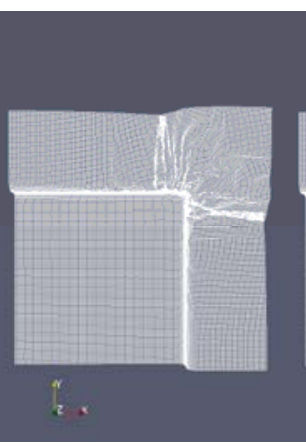

With shear without bending

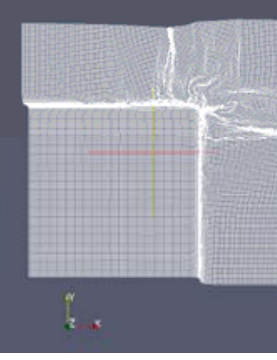

With shear with bending

Fig 12: Simulation results of square box deep drawing test

\section{Conclusions}

We proposed a model for the woven composite forming simulation. We presented a model to describe the woven's tensile behavior, shear behavior and the bending behavior. Compared with the tensile stiffness, shear stiffness and bending stiffness are actually very small for most textile fabrics. When some wrinkles appear, however, the model with the shear stiffness yields better results than those based on tensile properties only. And the result of the model with bending stiffness show that bending stiffness changes the wrinkle form. Based on the current model, the future work is to develop a model for the woven composite forming simulation to consider also the behavior of resin.

\section{Acknowledgement}

The authors would like to acknowledge the financial support to this work by the AIRCELLE/SAFRAN.

\section{References}

[1] O. K. Bergsm, J. Huisman, Deep drawing of fabric reinforced thermoplastics. In $2^{\text {nd }}$ conference on computer aided design in composite material technology, P. 323333, 1988.

[2] V. Der, F. Ween, Algorithms for draping fabrics on doubly curved surfaces. Int J Numer Method Eng, P. 1414-1426, 1991.

[3] X. Q. Peng, J. Cao, A continuum mechanics-based nonorthogonal constitutive model for woven composite fabrics, Composites Part A, Vol. 36, P. 859-874, 2005.

[4] W. R .Yu, F. Pourboghrat, Non-orthogonal constitutive equation for woven fabric reinforced thermoplastic composites. Composites Part A, P. 1095-1105, 2002.

[5] B. V. West, R. B. Pipes, M. Keefe, A simulation of the draping of bidirectional fabrics over arbitrary surfaces. J Text Inst, P. 448-460, 1990.

[6] P. Boisse, M. Borr, K. Buet, A. Cherouat, Finite element simulations of textile composite forming including the biaxial fabric behavior. Composites Part B, P. 453-464, 1997.

[7] N. Hamila, P. Boisse, Simulations of textile composite reinforcement draping using a new semi-discrete three node finite element. Composites Part B, P. 999-1010, 2008.

[8] B. Zouari, J. L. Daniel, P. Boisse, A woven reinforcement forming simulation method influence of the shear stiffness. Computers and Structures, P. 351-363, 2006.

[9] Q. Q. Chen, P. Boisse, N. Hamila, A. Saouab, J. Bréard, C. H. Park, A finite element method for the forming simulation of the reinforcements of thermoplastic composite. In ESAFORM 2009. 